J. clin. Invest. 34, 131 (1955). - 19. Kattus, A. A., R. Watanabe, Ch. Skmenson, W. Drell und C. M. Agress, J. amer. med. Assoc. 160, 16 (1956). - 20. KraUSe, S. und G. KraUSe, J. amer. med. Assoc. 161, 144 (1956). - 21. La Due, J. S., F. Wrobldwsski und A. Karmen, Science 120, 497 (1954). - 22. La Due, J. S. und F. Wroblewskr, Circulation 11, 871 (1955). - 23. La DUE, J. S., J. Nydick und F. Wroblewski, Circulation 12, 736 (1955). - 24. Merill, J. M., J. Lemley-Stone, J. T. Grace und G. R. Meneely, J. amer. med. Assoc. 160, 1454 (1956). - 25. Meyerhof, O. und K. Lohmand, Biochem. Z. 271, 89 (1934). - 26. Mold, A., Arch. Kreislaufforschg. 18, 210 (1952). - 27. Myers, G. B., H. A. Kletin, B. E. Stofer und T. HiratzKa, Amer. Heart J. 34, 785 (1947). 28. Myers, G. B. und H. A. Kletn, Amer. Heart J. 35, 727 (1948). - 29. Myers, G. B., H. A. Klein und T. Hiratzka, Amer. Heart J. 36, 838 (1948) und 38, 837 (1949). 30. Nypick, J., F. Wroblewski und J. S. La Due, Circulation 12, 161 (1955). - 31. Ostrow, B. H., D. Steminberg, H. E. Troktin und J. M. Evans, Circulatir- 12, 756 (1955). - 32. ReTNDelr, H. und H. KLEPZIG, Z. Kreislaufforschg. 39, 705 (1950). 33. Rudolph, L. A., R. Dutron und J. A. Schaefer, J. clin. Invest. 34, 960 (1955). 34. Schlitef, H. und P. Kaym, Klin. Wschr. 35, 1083 (1957). - 35. Schnetder, K. W., Z. klin. Med. 154, 525 (1957). - 36. Sibley, J. A. und A. L. LeHninger, J. biol. Chem. 177, 859 (1949). - 37. Spang, K. und A. WeLsCH, Dtsch. med. Wschr. 75, 849 (1950). 38. Stradss, H. und L. N. Katz, Amer. Heart J. 10, 546 (1935). - 39. Volk, B. W., S. Losner, St. M. Aronson und H. LeW, Amer. J. med. Sci. 292, 38 (1956). - 40. WARburg, O. und W. Christran, Biochem. Z. 814, 399 (1943). - 4l. Wollheim, E., Z. klin. Med. 108, 248 (1928). — 42. WollmetM, E., Z. klin. Med. 116, 269 (1931). — 43. WolLHEIM, E., Dtsch. med. Wschr. 14, 1 (1930). - 44. WollheIm, E., Verh. Dtsch. Ges. Inn. Med. 16, 75 (1950). - 45. Wollheim, E., Cardiologia 20, 327 (1952). - 46. Wollmeim, E., Klin. Wschr. 1955, 1065. - 47. WoldaheiM, E., Dtsch. med. Wschr. 81, 2080 (1956). 48. Wollmeim, E., und K. W. SchneIder, Verh. Dtsch. Ges. Inn. Med. 1954, 333. 49. Wolryeim, E. und K. W. Sohneider, Medizinische 26, 958 (1955). - 50. Wollheim, E., K. W. Schnerder und J. Zissler, CardiologenkongreB (Stockholm 1956). - 51. WroBLewski, F. und J. S. LA DUE, Cancer 8, 1155 (1955). - 52. Wroblewski, F. und J. S. LA Dok, J. amer. Assoc. 160, 1130 (1956).

Angchriften der Verfasser:

Dr. B. Brtbiahm, Priv.-Doz. Dr. K. W. Schnetder und Priv.-Doz. Dr. F. ParR, Med. Üniv.-Klinlk Wärzburg

\title{
Berichtigung
}

zur Arbeit

\section{Uber die Wirkungsweise der intraarteriellen Gasinsufflation auf den peripheren Kreislauf und ihre Gefahren}

\author{
Von G. Somönbach u. W. Thorban
}

$$
\text { Arch. Kreislaufforschg., 26, 97-112 (1957). }
$$

Auf S. 108, Zeile 33 muß es heißen statt: auf das 7.800 fache des Ausgangswertes, auf 7-800\% des Ausgangswertes oder das 7-8 fache des Ausgangswertes. 